\title{
Serum Podocalyxin Levels as High Risk Markers for CVDs of Complication of Diabetes
}

\author{
Hussein DhaherAyyez Al-fatlawiy ${ }^{1}$, Hanaa Addai Ali \\ ${ }^{1}$ Post Graduate, ${ }^{2}$ Prof. Dr., Department of Chemistry, College of Science, University of Kufa, Iraq
}

\begin{abstract}
Diabetes mellitus is a group of metabolic diseases characterized by hyperglycemia resulting from defects in insulin secretion, insulin action, or both. The chronic hyperglycemia of diabetes is associated with long-term damage, dysfunction, and failure of various organs, especially the eyes, kidneys, nerves, heart, and blood vessels; Podocalyxin is the major sialoprotein in the glycocalyx of glomerular podocytes, podocalyxin was found in the blood vessels of several organs.
\end{abstract}

Objective: The aim of the present study was to elevate serum podocalyxin and some biochemical parameters in diabetic patients compare with healthy group.

Method: The present case-control study included 90 participants divided into two groups: 60 patients with diabetes mellitus ( 40 males and 20 females; aged $49.87 \pm 8.089$ yr.) study group and 30 (20 males and 10 females; aged 48.27 \pm 6.335 yr.) a healthy group (control group). Podocalyxin (PODXL) and insulin measured using the ELISA kit. Anthropometric were measured fasting serum glucose levels and lipid profile were determined by photometric method.

Result: The mean serum podocalyxin levels was significantly increase in patients with diabetes mellitus Vs. healthy control group $(26.24 \pm 2.11 \mathrm{ng} \backslash \mathrm{ml}$ Vs. $17.45 \pm 1.57 \mathrm{ng} \backslash \mathrm{ml}$ respectively, $\mathrm{p}<0.001)$, BMI was significantly increased in patients diabetes mellitus Vs. healthy control group (29.766 \pm 3.225 Vs. $23.756 \pm 0.77$ respectively, $\mathrm{p}<0.001$ ) also the mean fasting insulin level was higher in patients with diabetes mellitus than the healthy control group $(7.23 \pm 1.26$ Vs. $5.05 \pm 1.14$ respectively, $\mathrm{p}<0.001)$, HOMA-IR significantly increased in patients with diabetes mellitus Vs. healthy control group ( $2.65 \pm 0.77$ Vs. $1.16 \pm 0.25$ respectively, $p<0.001)$ but the mean of HOMA- $\beta$ in patients with diabetes mellitus was significantly lower than the healthy control group (35.30 \pm 13.83 Vs. $61.91 \pm 19.87$ respectively, $\mathrm{p}<0.001$ ), lipid profile include TG, TC, LDL-C, VLDL-C significant increased in patients with diabetes mellitus compared with healthy control group $(211.50 \pm 88.02$ Vs. $116.87 \pm 38.43, \mathrm{p}<001),(240.13 \pm 56.20$ Vs. $141.43 \pm 33.34, \mathrm{p}<0.001),(161.87 \pm 56.39$ Vs. $65.82 \pm 30.97$, $\mathrm{p}<0.001),(42.30 \pm 17.60$ Vs. $23.37 \pm 7.68, \mathrm{p}<0.001)$ respectively except HDL-C were significantly decreased in patients with diabetes mellitus than the healthy control group (33.83 \pm 9.02 Vs. $52.23 \pm 7.08$ respectively, $\mathrm{p}<0.001$ ), Patients with diabetic mellitus have significantly higher serum podocalyxin levels than the controls. Podocalyxin levels positively significantly correlated with BMI, FSG, Insulin, HbA1c and HOMAIR levels while HOMO- $\beta$ significant negative correlation respectively with podocalyxin levels. podocalyxin may be a promising biomarker in patients with diabetes mellitus.

Conclusion: Podocalyxin may be a promising biomarker in patients with diabetes mellitus and can be used as a high risk markers for CVDs in DM patients.

Keywords: Diabetes mellitus, glycaemiaindices, insulinresistance, podocalyxin.

\section{Introduction}

Diabetes mellitus (DM) is a hyperglycemic, multifactor metabolic disease characterized by insulin secretion deficiencies, insulin activity, or both. Chronic hyperglycemia is one of the long-term, widespread diseases worldwide. This is linked with long-term microvascular and macrovascular diseases ${ }^{(1)}$. In addition to these serious medical conditions, however, other 
co-morbidities, such as retinopathy, nephropathy and cardiovascular disease also arise in patients with T2D over time $^{(2)}$.Symptoms of diabetes are normal in patients with Type 1 and type 2 diabetes, though at the same time causing substantial morbidity. The recurrent diabetes risks are primarily classified into microvascular and macrovascular, the former being far more common than the latter ${ }^{(3)}$. Neuropathy, nephropathy and retinopathy are the microvascular complications, while coronary and peripheral artery disorders have macrovascular complications $^{(3)}$.

Podocalyxin is the major sialoprotein in the glycocalyx of glomerular podocytes, podocalyxin. was found in the blood vessels of several organs (lung, heart, kidney, small intestine, brain, pancreas, aorta, the periportal blood vessels in the liver, and the central arteries of follicles of the spleen, but not in the endothelial that line the sinusoids of the latter organs) ${ }^{(4)}$. Podocalyxin (Podxl) is a sialomucin CD34 that is a major cell surface component that is expressed within the epithelial cells (podocyte) of the kidney glomerulus as a glycocalyx and was for- merly known as sialylated protein ${ }^{(5)}$.]. Podxl maintains the slit diaphragm and podocytes' shape ${ }^{(6)}$. When podocytes are injured, Podxl is released from the vesicle-like structures or microvilli and excreted in urine ${ }^{(7,8)}$ Renal Podxl is used as an early marker for diabetic nephropathy and is considered a biomarker for glomerular disease ${ }^{(9,10)}$. Podxl also has a wide expression on endothelial cell surface all over the body ${ }^{(11)}$ Podxl is expressed within neurons ${ }^{(13)}$ mesothelial cells that line organs, hematopoietic stem cells, ${ }^{(14)}$ megakaryocytes, and vascular endothelial cells. ${ }^{(12)}$ A recent study showed that serum Podxl (s-Podxl) levels were correlated with carotid intimal medial thickness ${ }^{(15)}$.

\section{Materials and Method}

Subject: In this case-control study (90) participated were recruited from AL-Najaf province- Iraq where divided into two groups: 60 patients with diabetes mellitus (40 males and 20 females) (study group) and 30 healthy control group (20 males and 10 females) thevisited patient DM at Diabetes and Endocrine Center Al-Sadr Medical City from 2019 December to February 2020. All of the 37-65 yr. without another chronic disease for example, immune system, Cardiovascular, thyroid, smoking, pregnant, patients with acute condition or complications including renal, hepatic, neurologic and pulmonary disease were excluded.
Blood Sample Collection: Five milliliters of venous blood were drawn from each the patients and control groups by medical syringes, and $2 \mathrm{ml}$ was but into EDTA tubes in order to used for $\mathrm{HbA1C}$ and the remaining the blood were placed in gel tubes and then left at room temperature for a period of ten minutes to fifteen minutes for coagulation, then centrifuged (at $3000 \mathrm{X} \mathrm{g}$ ) for 10 minutes for serum delivery. The sera were separated into four Eppendorftubes and stored at $\left(-20 C^{\circ}\right)$ until time of biochemical estimation.

Anthropometric and Biochemical study: Demographic characteristic were measured and included waist, hip, height $(\mathrm{cm})$, utilizing a standardized measuring tape in $\mathrm{cm}$, weight $(\mathrm{Kg})$ and BMI (calculated $\mathrm{a} \mathrm{Kg} \backslash \mathrm{m}^{2}$ )

Fasting analysis serum glucose, lipid profile level were measured by enzymatic method. The concentration of fasting insulin and podocalyxinwere determined by ELISA kits (MLSIN kit, china), (MLSIN kit, china) respectively $\mathrm{HbA1c}$ (the CLOVER A1c Self analyzer is an IVD (In Vitro Diagnostic Device), insulin resistance index (Homeostatic model assessment-insulin resistance). HOMA-IR $=$ [glucose $(\mathrm{mg} \backslash \mathrm{dl}) \mathrm{X}$ Insulin $(\mu \mathrm{U} / \mathrm{ml})] / 405$ cutoff value of HOMA-IR is $>2.5^{(16)}$, HOMA- $\beta \%=360 x$ Insulin/(Glucose -63$)^{(17)}$.

The body mass index (BMI) was calculated at the ratio of weight in $(\mathrm{Kg})$ to the height squared $\left(\mathrm{m}^{2}\right)^{(18)}$.

Statistical Method: Statistical evaluation was done by SPSS version 22 (IBM, Inc) and included a univariate and multivariate, multinominal, linear, and logistic regression analysis, Wallis test, chi-square test, and independent-sample Student t-test as appropriate. The data was shown as the mean \pm standard deviation or as a number with a percentage for categorical variables. A change in $\beta$ of $>10 \%$ was used to identify confounding in multivariate analysis.

\section{Result and Discussion}

As shown table (1) was illustrated the general characteristics of the study this includes data of patients with diabetes and a healthy group.

The level of fasting serum glucose, $\mathrm{HbA1C}$, insulin, HOMA-IR were significantly higher $(p<0.001)$ compared to the healthy group while the data of HOMA- $\beta$ decreased in patients DM group. 
Podocalyxin level was significant $(\mathrm{p}<0.001)$ higher in patient with DM group $(26.24 \pm 2.11 \mathrm{ng} \backslash \mathrm{ml})$ and healthy group (17.45 $\pm 1.57 \mathrm{ng} \backslash \mathrm{ml})$.

The present results shown in table (2) the correlation between levels of podocalyxin and the biochemical studied in patients with diabetes mellitus.

HDL-C have a non-significant negative correlations, $(-0.095)$, respectively with podocalyxin levels.
Age, waist, hip, HbA1c, TG-C, TC, LDL-C, VLDL-C non-significant positive correlation(0.209-), (0.119), (0.153-), (0.022), (0.044), (0112), (0.254), $(0.044)$ respectively with podocalyxin levels.

HOMO- $\beta$ significant negative correlation (-0.122-) respectively with podocalyxin levels.

While BMI, FSG, Insulin and HOMA-IR asignificant positive correlations (0.353), (0.168), (0.136) respectively with podocalyxin level in DM patients.

Table (1): Characteristics and differences between DM patients group and healthy group.

\begin{tabular}{|c|c|c|c|}
\hline Parameters & $\begin{array}{c}\text { DM Patients group } \\
\text { Mean } \pm \text { SD }\end{array}$ & $\begin{array}{l}\text { Healthy group } \\
\text { Mean } \pm \text { SD }\end{array}$ & P-value \\
\hline Sex. $(M \backslash F)$ & $60(40 \backslash 20)$ & $30(20 \backslash 10)$ & - \\
\hline Age (Years) & $49.87 \pm 8.08$ & $48.27 \pm 6.33$ & $0.437 \mathrm{NS}$ \\
\hline $\mathrm{BMI}\left(\mathrm{Kg} \backslash \mathrm{m}^{2}\right)$ & $29.76 \pm 3.22$ & $23.75 \pm 0.77$ & $\leq 0.001$ \\
\hline $\mathrm{WC}(\mathrm{cm})$ & $109.66 \pm 10.84$ & $98.86 \pm 10.82$ & $\leq 0.001$ \\
\hline Hip (cm) & $110.70 \pm 16.37$ & $99.23 \pm 11.46$ & $\leq 0.001$ \\
\hline WHR & $1.00 \pm 0.14$ & $0.99 \pm 0.03$ & $0.237 \mathrm{NS}$ \\
\hline $\mathrm{FSG}(\mathrm{mg} \backslash \mathrm{dL})$ & $147.27 \pm 31.52$ & $94.13 \pm 8.26$ & $\leq 0.001$ \\
\hline Insulin $(\mu \mathrm{UI} \backslash \mathrm{mL})$ & $7.23 \pm 1.26$ & $5.05 \pm 1.14$ & $\leq 0.001$ \\
\hline $\mathrm{HbA} 1 \mathrm{C} \%$ & $7.73 \% \pm 1.68 \%$ & $4.02 \% \pm 0.52 \%$ & $\leq 0.001$ \\
\hline HOMA-IR & $2.65 \pm 0.77$ & $1.16 \pm 0.25$ & $\leq 0.001$ \\
\hline НОМА- $\beta$ & $35.30 \pm 13.83$ & $61.91 \pm 19.87$ & $\leq 0.001$ \\
\hline $\mathrm{TG}(\mathrm{mg} \backslash \mathrm{dL})$ & $211.50 \pm 88.02$ & $116.87 \pm 38.43$ & $\leq 0.001$ \\
\hline $\mathrm{TC}(\mathrm{mg} \backslash \mathrm{dL})$ & $240.13 \pm 56.20$ & $141.43 \pm 33.34$ & $\leq 0.001$ \\
\hline LDL-C (mg\dL) & $161.87 \pm 56.39$ & $65.82 \pm 30.97$ & $\leq 0.001$ \\
\hline VLDL-C (mg\dL) & $42.30 \pm 17.60$ & $23.37 \pm 7.68$ & $\leq 0.001$ \\
\hline HDL-C (mg\dL) & $33.83 \pm 9.02$ & $52.23 \pm 7.08$ & $\leq 0.001$ \\
\hline Podocalyxin (ng\mL) & $26.24 \pm 2.11$ & $17.45 \pm 1.57$ & $\leq 0.001$ \\
\hline
\end{tabular}

Data presented as Mean $\pm \mathrm{SD}$, standard deviation, BMI: body mass index, WHR: ratio of waist to hip, WC: waist circumference, FSG: Fasting serum glucose, HbA1C: Glycated hemoglobin, SD: Standard deviation, significant difference at $(\mathrm{p}<0.05), \mathrm{TC}$ : Total cholesterol,
HDL-C: High density lipoprotein- Cholesterol, LDL-C: Low density lipoprotein- Cholesterol, VLDL-C: Very low density lipoprotein-Cholesterol, TG: Triglycerides, significant at $(\mathrm{P}<0.01)$. 


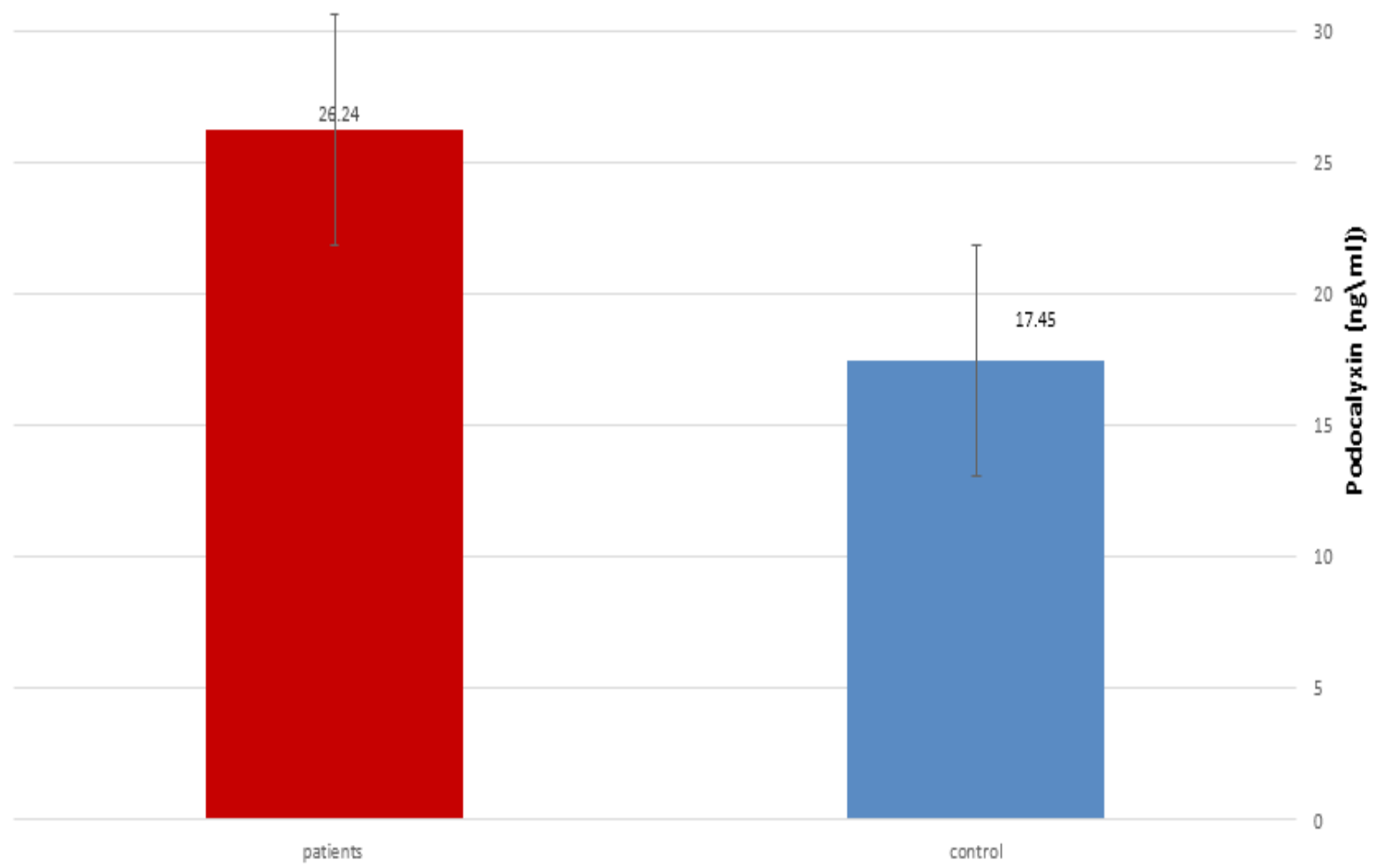

Figure (1): Comparison of mean podocalyxin level between control and patients group.

Table (2): Correlation between level of podocalyxin and studied parameters in DM patients group

\begin{tabular}{|l|c|c|}
\hline Parameters & $\mathbf{r}$ & p-value \\
\hline Age $($ years $)$ & $0.209-$ & 0.26 \\
\hline BMI $\left(\mathrm{kg} \backslash \mathrm{m}^{2}\right)$ & 0.353 & $0.05^{*}$ \\
\hline WC $(\mathrm{cm})$ & 0.119 & $0.05^{*}$ \\
\hline Hip $(\mathrm{cm})$ & $0.135-$ & 0.47 \\
\hline WHR & 0.223 & $0.02^{*}$ \\
\hline FSG $(\mathrm{mg} \backslash \mathrm{dL})$ & 0.186 & $0.03^{*}$ \\
\hline Insulin $(\mu \mathrm{U} / \mathrm{mL})$ & 0.136 & $0.04^{*}$ \\
\hline HbA1C\% & $0.022-$ & $0.059^{*}$ \\
\hline HOMA-IR & 0.242 & $0.01^{*}$ \\
\hline HOMA- $\beta$ & $-0.122-$ & $0.03^{*}$ \\
\hline HDL-C $(\mathrm{mg} \backslash \mathrm{dL})$ & -0.095 & 0.61 \\
\hline TG-C $(\mathrm{mg} \backslash \mathrm{dL})$ & 0.044 & 0.81 \\
\hline TC $(\mathrm{mg} \backslash \mathrm{dL})$ & 0.112 & 0.058 \\
\hline LDL-C $(\mathrm{mg} \backslash \mathrm{dL})$ & 0.254 & 0.175 \\
\hline VLDL-C $(\mathrm{mg} \backslash \mathrm{dL})$ & 0.044 & 0.818 \\
\hline
\end{tabular}

BMI: body mass index, FSG: fasting serum glucose HOMA-IR: hemostasis model assessment-insulin resistance assessment- HOMA- $\beta \%$ : hemostasis model assessment-beta cell percentage. TG: triglyceride, HDL-C: High-density lipoprotein-cholesterol, LDL-C: low density lipoprotein-cholesterol. Data represented as Mean $\pm \mathrm{SD}$ : standard deviation, $\mathrm{NS}=$ non- significant differences at $(\mathrm{P}>0.05) . *=$ significant differences at $(\mathrm{P} \leq$ $0.05),{ }^{* *}=$ significant differences at $(\mathrm{P} \leq 0.01)$

Insulin resistance and $\mathrm{T} 2 \mathrm{DM}$ are characterized by dyslipidemia one major risk factor for cardiovascular disease $^{(19)}$ comprise with hypertriglyceridemia, low levels of high-density lipoprotein cholesterol (HDL-C) and the appearance of small, dense, LDL (sdLDL) and caused excessive postprandial lipemia ${ }^{(20)}$ Diabetic dyslipidemia caused from the disturbance of lipid metabolism, an early event cardiovascular complications development and was preceded in T2DM patients by several years. ${ }^{(21)}$ Lipid abnormalities in patients with diabetes often termed "diabetic dyslipidemia", are typically characterized by high total cholesterol 
(T-Chol), high triglycerides (TG), low high-density lipoprotein cholesterol (HDL-C) and increased levels of small dense (LDL-C) particles. Low-density lipoprotein cholesterol (LDL-C) levels may be moderately increased or normal ${ }^{(22)}$. Podocalyxin is strongly represented by glomerular podocytes, endothelium vascular and hematopoietic cells ${ }^{(10)}$. Showed that s-Podxl levels were significantly higher in patients with diabetic patients compared with healthy group. A recent study reported an association between s-Podxl concentrations and markers of CVD.Shoji et.al claimed that s-Podxl levels were significantly associated with cIMT and this association remained significant even after controlling the common CVD risk factors such as diabetes, dyslipidemia, hypertension, sex, age and current smoker ${ }^{(23)}$. In other study illustrated, the number of cases with diabetic and CHD increased with increasing tertiles of s- Podxl from $11(15 \%)$ in the 1 st tertile, over $22(30 \%)$ in the 2 nd tertile, to $25(33 \%)$ in the 3 rd tertile ${ }^{(24)}$.

Previous study showed that the re- lease of Podxl from injured podocytes occurs as vesicle-like struc- tures and/or as a result of shedding microvilli ${ }^{(25)}$.Various types of cells and tissues express Podxl including neurons, lungs, platelets and vascular en- dothelial cells ${ }^{(26)}$.Our results of high s-Podxl levels in patients with DM might be due to its release from injured endothelium through amechanism, which may be similar to that of podxl release from in- juredpodoctyes.Previous studies have shown the role of Podxl in endo- thelial function and vascular inflammation ${ }^{(27)}$. Another study showed that, there was an increase in non- specific inflammatory infiltrates within the vessels and CRP level in mu- rine endothelial cells after conditional knock out of the Podxl gene ${ }^{(28)}$.In our study, we found higher levels of s-Podxl in patients with DM com- pared with healthy group that s-Podxl might be associ- ated with CVD independently of diabetes ${ }^{(29)}$.

\section{Conclusion}

Podocalyxin levels positively correlated with BMI, FSG, insulin, and HOMA-IR levels while HOMA- $\beta$ significantly negative correlation respectively with podocalyxin levels. podocalyxin may be a promising biomarker in patients with diabetes mellitus .Podxl can be used either alone or with other markers for cardiovascular disease to determine diabetic patients with high risk of complication diabetes.

Acknowledgements: I would like to present special thanks to my supervisor prof. Dr. Hanaa Addai Ali for her, suggested this research continuous advice, guidance and encouragement. The authors also would like to thank the volunteers from the Diabetes and Endocrine Centre in Al-Sadr Medical City AL-Najaf province for the recruitment of subjects.

Ethical Clearance: The Research Ethical Committee at scientific research by ethical approval of both $\mathrm{MOH}$ and MOHSER in Iraq.

\section{Conflict of Interest: None}

Funding: Self-funding

\section{Reference}

1. Yimer EM, Zewdie KA, Hishe HZ. Review Article Netrin as a Novel Biomarker and Its Therapeutic Implications in Diabetes Mellitus and DiabetesAssociated Complications. 2018;2018:5-7.

2. Association AD. Diagnosis and classification of diabetes mellitus. Diabetes Care. 2009;32(Supplement 1):S62-S67.

3. Deshpande AD, Harris-Hayes M, Schootman M. Epidemiology of diabetes and diabetes-related complications. Phys Ther. 2008;88(11):1254-1264.

4. Horvat R, Hovorka A, Dekan G, Poczewski $\mathrm{H}$, Kerjaschki D. Endothelial cell membranes contain podocalyxin--the major sialoprotein of visceral glomerular epithelial cells. J Cell Biol. 1986;102(2):484-491.

5. Kerjaschki D, Sharkey DJ, Farquhar MG. Identification and characterization of podocalyxin-the major sialoprotein of the renal glomerular epithelial cell. J Cell Biol. 1984;98(4):1591-1596.

6. Doyonnas R, Kershaw DB, Duhme C. Anuria, omphalocele, and perinatal lethality in mice lacking the CD34-related protein podocalyxin. J Exp Med. 2001;194(1):13-28.

7. Takeda T, McQuistan T, Orlando RA, Farquhar MG. Loss of glomerular foot processes is associated with uncoupling of podocalyxin from the actin cytoskeleton. J Clin Invest. 2001;108(2):289-301.

8. Hara M, Yamamoto $\mathrm{T}$, Yanagihara T. Urinary excretion of podocalyxin indicates glomerular epithelial cell injuries in glomerulonephritis. Nephron. 1995;69(4):397-403.

9. Hara M, Yamagata K, Tomino Y, et al. Urinary podocalyxin is an early marker for podocyte injury 
in patients with diabetes: establishment of a highly sensitive ELISA to detect urinary podocalyxin. Diabetologia. 2012;55(11):2913-2919.

10. Shoji M, Kobayashi K, Takemoto M, Sato Y, Yokote $\mathrm{K}$. Urinary podocalyxin levels were associated with urinary albumin levels among patients with diabetes. Biomarkers. 2016;21(2):164-167.

11. Chen Q, Wang Y, Li Y, Zhao M, Nie G. Serum podocalyxin is significantly increased in early-onset preeclampsia and may represent a novel marker of maternal endothelial cell dysfunction. J Hypertens. 2017;35(11):2287-2294.

12. Horvat R, Hovorka A, Dekan G, Poczewski H, Kerjaschki D. Endothelial cell membranes contain podocalyxin--the major sialoprotein of visceral glomerular epithelial cells. J Cell Biol. 1986;102(2):484-491.

13. Vitureira N, Andrés R, Pérez-Martínez E. Podocalyxin is a novel polysialylated neural adhesion protein with multiple roles in neural development and synapse formation. PLoS One. 2010;5(8):e12003.

14. Doyonnas R, Nielsen JS, Chelliah S. Podocalyxin is a CD34-related marker of murine hematopoietic stem cells and embryonic erythroid cells. Blood. 2005;105(11):4170-4178.

15. Shoji M, Takemoto M, Kobayashi K.Serum podocalyxin levels correlate with carotid intima media thickness, implicating its role as a novel biomarker for atherosclerosis. Sci Rep. 2018;8(1):17.

16. Pennings N, Jaber J, Ahiawodzi P. Ten-year weight gain is associated with elevated fasting insulin levels and precedes glucose elevation. Diabetes Metab Res Rev. 2018;34(4):e2986.

17. DeUgarte CM, Bartolucci AA, Azziz R. Prevalence of insulin resistance in the polycystic ovary syndrome using the homeostasis model assessment. Fertil Steril. 2005;83(5):1454-1460.

18. Nuttall FQ. Body mass index: Obesity, BMI, and health: A critical review. Nutr Today. 2015;50(3):117-128.

19. Grundy SM. Hypertriglyceridemia, atherogenic dyslipidemia, and the metabolic syndrome. Am J Cardiol. 1998;81(4):18B-25B.
20. Ginsberg HN, Zhang Y-L, Hernandez-Ono A. Metabolic syndrome: focus on dyslipidemia. Obesity. 2006;14(2S):41S.

21. Adiels M, Olofsson S-O, Taskinen M-R, Borén J. Diabetic dyslipidaemia. Curr Opin Lipidol. 2006;17(3):238-246.

22. Mooradian AD. Dyslipidemia in type 2 diabetes mellitus. Nat Rev Endocrinol. 2009;5(3):150-159.

23. Shoji M, Takemoto M, Kobayashi K, et al. Serum podocalyxin levels correlate with carotid intima media thickness, implicating its role as a novel biomarker for atherosclerosis. Sci Rep. 2018;8(1):17.

24. El-ashmawy HM, Selim FO, Hosny TAM, Almassry HN. Journal of Diabetes and Its Complications Association of serum podocalyxin levels with peripheral arterial disease in patients with type 2 diabetes. J Diabetes Complications. 2019;33(7):495-499.

25. Hara M, Yanagihara T, Hirayama Y. Podocyte membrane vesicles in urine originate from tip vesiculation of podocyte microvilli. Hum Pathol. 2010;41(9):1265-1275.

26. Wang J, Zhao Y, Qi R. Prognostic role of podocalyxin-like protein expression in various cancers: A systematic review and meta-analysis. Oncotarget. 2017;8(32):52457.

27. Debruin EJ, Hughes MR, Sina C.Podocalyxin regulates murine lung vascular permeability by altering endothelial cell adhesion. PLoS One. 2014;9(10).

28. Horrillo A, Porras G, Ayuso MS, GonzálezManchón C. Loss of endothelial barrier integrity in mice with conditional ablation of podocalyxin (Podxl) in endothelial cells. Eur J Cell Biol. 2016;95(8):265-276.

29. El-ashmawy HM, Selim FO, Hosny TAM, Almassry HN. Journal of Diabetes and Its Complications Association of serum podocalyxin levels with peripheral arterial disease in patients with type 2 diabetes. J Diabetes Complications. 2019;33(7):495-499. 